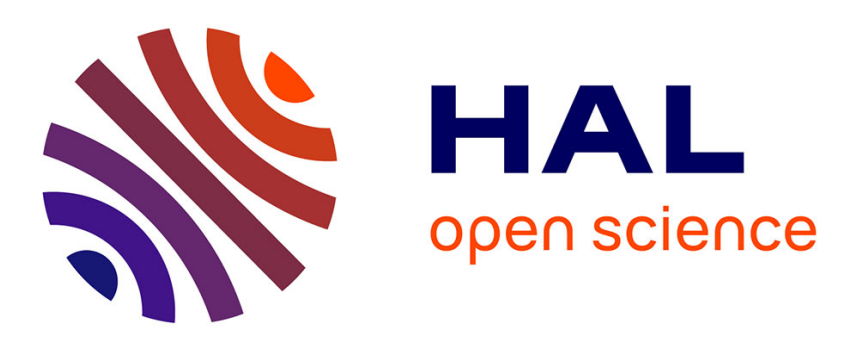

\title{
Building Generalized Open Boundary Conditions for Fluid Dynamics Problems
}

Eric Blayo, Véronique Martin

\section{To cite this version:}

Eric Blayo, Véronique Martin. Building Generalized Open Boundary Conditions for Fluid Dynamics Problems. International Journal for Numerical Methods in Fluids, 2013, 71 (4), pp.506-521. 10.1002/fld.3675 . hal-00685195

\section{HAL Id: hal-00685195 https://hal.science/hal-00685195}

Submitted on 4 Apr 2012

HAL is a multi-disciplinary open access archive for the deposit and dissemination of scientific research documents, whether they are published or not. The documents may come from teaching and research institutions in France or abroad, or from public or private research centers.
L'archive ouverte pluridisciplinaire HAL, est destinée au dépôt et à la diffusion de documents scientifiques de niveau recherche, publiés ou non, émanant des établissements d'enseignement et de recherche français ou étrangers, des laboratoires publics ou privés. 


\title{
Building Generalized Open Boundary Conditions for Fluid Dynamics Problems
}

\author{
Eric Blayo, Véronique Martin,

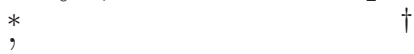

April 4, 2012

\begin{abstract}
This paper deals with the design of an efficient open boundary condition (OBC) for fluid dynamics problems. Such problematics arise, for instance, when one solves a local model on a fine grid that is nested in a coarser one of greater extent. Usually the local solution $U^{\text {loc }}$ is computed from the coarse solution $U^{\text {ext }}$ thanks to an OBC formulated as $B_{h} U^{\text {loc }}=B_{H} U^{\text {ext }}$, where $B_{h}$ and $B_{H}$ are discretizations of the same differential operator $\mathcal{B}\left(B_{h}\right.$ being defined on the fine grid and $B_{H}$ on the coarse grid). In this paper we show that such an OBC cannot lead to the exact solution, and we propose a generalized formulation $B_{h} U^{\text {loc }}=B_{H} U^{\text {ext }}+g$, where $g$ is a correction term. When $B_{h}$ and $B_{H}$ are discretizations of a transparent operator, $g$ can be computed analytically, at least for simple equations. Otherwise we propose to approximate $g$ by a Richardson extrapolation procedure. Numerical test cases on a 1-D Laplace equation and on a 1-D shallow water system illustrate the improved efficiency of such a generalized OBC compared to usual ones.
\end{abstract}

Keywords : Open boundary conditions, nested grids, transparent boundary conditions, Richardson extrapolation, shallow water equations, fluid dynamics.

\section{Introduction}

In order to limit their computational cost, numerical simulations are frequently performed on a limited domain of interest which is only a portion of the full area in which the phenomenon takes place. This is the case, for instance, in a number of fluid dynamics applications, especially in geophysical ones, e.g. meteorology or oceanography. One then has to deal with the treatment of a fluid boundary artificially limiting the computational domain. This is the so called open boundary problem. The difficulty is then to prescribe open boundary conditions (OBCs) that are able both to evacuate the outgoing information and to take into account the incoming part of the available external information generally provided by some previous large scale low resolution simulation.

\footnotetext{
*Laboratoire Jean Kuntzmann, Université de Grenoble and INRIA, Eric.Blayo@imag.fr

†LAMFA UMR-CNRS 7352, Université de Picardie Jules Verne, 33 Rue St. Leu, 80039 Amiens, France veronique.martin@u-picardie.fr
} 
There exists a huge literature dealing with this problem in the context of various applications (see, e.g., the review papers $[2,7,8,19]$ ). In the specific context of ocean modelling, several kinds of OBCs have been proposed, such as damped radiation or Flather conditions (see, e.g., [3] and references therein for a review) and this question remains an active field of research (e.g. the recent papers $[9,13,14,15,16,17])$. As pointed out in [3], the conditions that seem to lead to the best numerical performances rely more or less directly on the mathematical notion of Transparent Boundary Conditions (TBCs). This is also consistent with several recent works that derive OBCs closely connected to TBCs (e.g. $[10,11,12,16,17]$ ). The problematic of TBC consists in finding an adequate boundary condition, such that solving $\mathcal{L} w^{\text {loc }}=\tilde{f}$ in $\Omega^{\text {loc }} \subset \Omega$ with this boundary condition leads to $w^{\text {loc }}=w_{\mid \Omega^{\text {loc }}}$, where $\mathcal{L} w=\tilde{f}$ in $\Omega$. In [5] transparent conditions are derived for several linear equations when the support of $\tilde{f}$ is included in $\Omega^{\text {loc }}$. Such conditions are used in actual OBC problems.

In this paper, we show that in the context of open boundary conditions, since the local problem is solved on a fine grid but uses exterior data that were obtained on a coarser grid (and/or even with a simplified physics), then the problem is related to a discrete TBC formulation with a right hand side $\tilde{f}$ which is non compactly supported in $\Omega^{\text {loc }}$. A consequence of this specific aspect is that an additional correction term is needed in the usual OBCs, in order to improve their efficiency (Section 2). In [1, 4] the case of $\tilde{f}$ non compactly supported in $\Omega^{\text {loc }}$ is treated in the discrete case. But in the context of OBCs, particular behaviors may appear, like for instance an oscillating behavior of the right hand side at the grid scale (see example below). As a consequence such r.h.s can not be considered on the coarse grid only. Our strategy is then to interpret the correction term at the continuous level which makes it possible to discretize it on the coarse exterior domain only (Section 3). We also propose in Section 4 another strategy to derive the correction term based on a Richardson extrapolation procedure. The efficiency of such generalized OBCs with regard to usual ones is assessed in the simple test cases of a 1-D Laplace equation and a 1-D shallow water system.

\section{Formal analysis of the open boundary prob- lem}

Let $\Omega$ be an open set in $\mathbb{R}^{n}$. We consider the following model:

$$
\begin{cases}\mathcal{L} u=f & \text { in } \Omega \\ \mathcal{C} u=g & \text { on } \partial \Omega\end{cases}
$$

where $\mathcal{L}$ is a (system of) partial differential equation(s), and $\mathcal{C}$ is a boundary operator. We are interested in obtaining an accurate approximation of the solution of this (system of) equation(s) in a local region included in $\Omega$. Ideally, if there were no limitations in terms of computer resources, one would first solve (1) accurately on the fine mesh $\Omega^{h}$ :

$$
\begin{cases}L_{h} U^{\mathrm{ref}}=f_{h} & \text { in } \Omega^{h} \\ C_{h} U^{\mathrm{ref}}=g_{h} & \text { on } \partial \Omega^{h}\end{cases}
$$

where $L_{h}$ and $C_{h}$ (resp. $f_{h}$ and $g_{h}$ ) are high resolution discretizations of $\mathcal{L}$ and $\mathcal{C}$ (resp. $f$ and $g$ ). Then we would focus on the restriction of $U^{\text {ref }}$ to the zone of 
interest $\Omega^{\text {loc }} \subset \Omega^{h}$ (see Figure 1). However such a global accurate computation is unaffordable. That is why one solves instead the local problem:

$$
\left\{\begin{array}{l}
L_{h} U^{\mathrm{loc}}=f_{h} \quad \text { in } \Omega^{\mathrm{loc}} \\
C_{h} U^{\mathrm{loc}}=g_{h} \quad \text { on } \partial \Omega^{\mathrm{loc}} \cap \partial \Omega^{h} \\
+ \text { a boundary condition at the open boundary } \Gamma
\end{array}\right.
$$

where $\Gamma$ is the artificial interface between $\Omega^{\text {loc }}$ and $\Omega^{h} \backslash \Omega^{\text {loc }}$.

The boundary condition that appears in (3) is called an open boundary condition (OBC) and should be ideally such that $U^{\text {loc }}$ coincides with the reference solution $U^{\text {ref }}$ in $\Omega^{\text {loc }}$. To this end, since the source term $f$ is not equal to zero in $\Omega \backslash \Omega^{\text {loc }}$, it is necessary to supply information on the behavior of $U^{\text {ref }}$ in this exterior region in the OBC. Since $U^{\text {ref }}$ is unknown, it is obvious that only approximate information is available. In the actual context of oceanic or atmospheric simulations, a coarse approximation $U^{\text {ext }}$ of $U^{\text {ref }}$ is generally available, either from some climatological database or from a previous coarse resolution calculation on $\Omega$. This coarse solution $U^{\text {ext }}$ will then be used in the OBC.
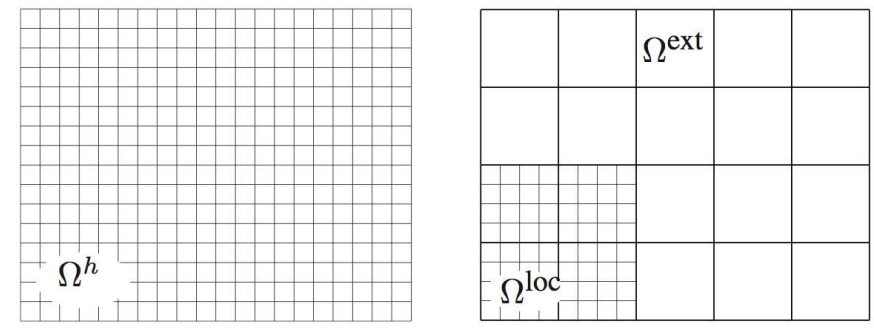

Figure 1: Example of configuration. Left: reference mesh $\Omega^{h}$; right: exterior coarse mesh $\Omega^{\text {ext }}$ and local fine mesh $\Omega^{\text {loc }}$.

Many existing OBCs are formulated under the usual form

$$
B_{h} U^{\mathrm{loc}}=B_{H} U^{\mathrm{ext}},
$$

where the boundary operators $B_{h}$ and $B_{H}$ are discretizations of the same differential operator $\mathcal{B}\left(B_{h}\right.$ being defined on the fine grid $\Omega^{\text {loc }}$ and $B_{H}$ on the coarse grid where $U^{\text {ext }}$ is available). For instance, the simplest choice is of course a Dirichlet condition $(\mathcal{B}=\mathrm{Id})$. Another example is the Flather condition [6], which is frequently used in oceanic and atmospheric modelling, and is generally quite efficient. As pointed out in [3], $\mathcal{B} u$ corresponds in this case to the incoming characteristic variable of the hyperbolic shallow water equations, and is an approximation of the exact transparent boundary condition (see, e.g., [5]). Let us now see if this usual form (4) can lead to the exact solution $U^{\text {loc }}=U_{\mid \Omega^{\text {loc }}}^{\text {ref }}$. We will assume in the following that every considered boundary operator $B_{h}$ is admissible, which means that solving (3) with the $\mathrm{OBC} B_{h} U^{\text {loc }}=z$ for any right hand side $z$, yields a unique solution. Moreover we will assume that we are in the simple case where all the operators $(\mathcal{L}, \mathcal{C}, \mathcal{B}$ and their discretizations) are linear.

By subtracting (3) from (2), let us write the equations for the error $U_{\mid \Omega^{l o c}}^{\mathrm{ref}}-$ 
$U^{\text {loc }:}$

$$
\begin{cases}L_{h}\left(U_{\mid \Omega^{\text {loc }}}^{\text {ref }}-U^{\text {loc }}\right)=0 & \text { in } \Omega^{\text {loc }} \\ C_{h}\left(U_{\mid \Omega^{\text {loc }}}^{\text {loc }}-U^{\text {loc }}\right)=0 & \text { on } \partial \Omega^{\text {loc }} \cap \partial \Omega^{h} \\ B_{h}\left(U^{\text {ref }}-U^{\text {loc }}\right)=B_{h} U^{\text {ref }}-B_{H} U^{\text {ext }} & \text { on } \Gamma .\end{cases}
$$

Since the problem is linear and $B_{h}$ is admissible, the solution is exact (i.e. $\left.U_{\mid \Omega^{l o c}}^{\mathrm{ref}}-U^{\text {loc }}=0\right)$ if and only if the right hand side of the OBC is zero. Unfortunately $B_{h} U^{\text {ref }}-B_{H} U^{\text {ext }}$, although probably small, is certainly not exactly zero. This means that, in the context of the usual form of OBC (4), it is not possible to obtain the exact solution, whatever the choices for $B_{h}$ and $B_{H}$.

We propose thus to introduce an additional term in the usual OBC, which leads to the generalized $O B C$ :

$$
B_{h} U^{\mathrm{loc}}=B_{H} U^{\mathrm{ext}}+g .
$$

In that case, the error satisfies the boundary condition $B_{h}\left(U^{\text {ref }}-U^{\text {loc }}\right)=$ $B_{h} U^{\text {ref }}-B_{H} U^{\text {ext }}-g$, in which the right hand side may vanish, provided that $g$ is well chosen. We have then proved the following lemma:

Lemma 1 If the two discrete operators $B_{h}$ and $B_{H}$ and the correction term $g$ are such that

$$
B_{h} U^{r e f}=B_{H} U^{e x t}+g
$$

then solving (3) with the generalized $O B C(6)$ provides the exact solution $U^{\text {loc }}=$ $U_{\mid \Omega^{l o c}}^{r e f}$.

Note that this property does not imply the uniqueness of the OBC: for each choice of $B_{h}$ and $B_{H}$, a convenient $g$ can be built (at least theoretically) such that (7) is satisfied.

In the rest of this paper, we will propose two different strategies to derive such a generalized $\mathrm{OBC}$, and implement them numerically in simple 1-D test cases.

\section{Generalized OBC based on Transparent Bound- ary Condition}

In this section we will illustrate how one can build a generalized $\mathrm{OBC}$ based on the use of a transparent boundary condition (TBC). Our strategy is to derive this TBC at the continuous level, and to discretize it afterwards. Since we intend here to make the derivation explicit, we will focus on the simple 1-D diffusion problem:

$$
\left\{\begin{array}{l}
\mathcal{L} u=-\frac{d^{2} u}{d x^{2}}+\alpha u=f \quad \text { in }(0,1) \\
u(0)=u(1)=0
\end{array}\right.
$$

with $\alpha$ a positive scalar, and $f$ in $C^{2}([0,1])$ (note that other linear boundary conditions could be considered without changing the main results of the paper). The generalization to more complex equations will be discussed in the conclusion. 


\subsection{Transparent boundary condition for an equation with a non local source term}

In Section 3.2 we will be confronted to the building of a TBC at $x=x_{\text {loc }}$ for a problem similar to $(8)$ when the support of the right hand side is not included in $\left(0, x_{\mathrm{loc}}\right)$. If it were, the derivation would follow the strategy presented in [5]. In the case of a right hand side non compactly supported in $\left(0, x_{\text {loc }}\right)$ TBCs have been derived $[1,4]$ in the discrete case. The following theorem is the continuous formulation of these last results.

Theorem 1 Let $w_{0}$ be a real number and $\tilde{f}$ be in $L^{2}\left(x_{l o c}, 1\right)$. If $w$ is the solution of

$$
\left\{\begin{array}{l}
\mathcal{L} w=\tilde{f} \text { in }\left(x_{l o c}, 1\right) \\
w\left(x_{l o c}\right)=w_{0} \\
w(1)=0
\end{array}\right.
$$

then $w$ satisfies the boundary condition:

$$
\mathcal{B} w:=w^{\prime}\left(x_{l o c}\right)+\lambda w\left(x_{l o c}\right)=p
$$

where $\lambda=-\frac{z^{\prime}\left(x_{l o c}\right)}{z\left(x_{l o c}\right)}, p=\frac{\int_{x_{l o c}}^{1} \tilde{f}(\sigma) z(\sigma) d \sigma}{z\left(x_{l o c}\right)}$ and $z(x)=e^{\sqrt{\alpha}(1-x)}-e^{-\sqrt{\alpha}(1-x)}$.

Proof: The exact expression of the solution $w$ can be obtained by using a decomposition $w=w_{1}+w_{2}$ with

$$
\left\{\begin{array} { l } 
{ \mathcal { L } w _ { 1 } = 0 \text { in } ( x _ { \mathrm { loc } } , 1 ) } \\
{ w _ { 1 } ( x _ { \mathrm { loc } } ) = w _ { 0 } } \\
{ w _ { 1 } ( 1 ) = 0 , }
\end{array} \quad \text { and } \quad \left\{\begin{array}{l}
\mathcal{L} w_{2}=\tilde{f} \text { in }\left(x_{\mathrm{loc}}, 1\right) \\
w_{2}\left(x_{\mathrm{loc}}\right)=0 \\
w_{2}(1)=0
\end{array}\right.\right.
$$

The solutions of these second order ODEs are $w_{1}(x)=w_{0} \frac{z(x)}{z\left(x_{\mathrm{loc}}\right)}$ and

$$
\begin{aligned}
w_{2}(x) & =-\frac{1}{2 \sqrt{\alpha}} \frac{z(x)}{z\left(x_{\mathrm{loc}}\right)} \int_{1}^{x \mathrm{loc}} \tilde{f}(\sigma)\left(e^{\sqrt{\alpha}\left(\sigma-x_{\mathrm{loc}}\right)}-e^{-\sqrt{\alpha}\left(\sigma-x_{\mathrm{loc}}\right)}\right) d \sigma \\
& +\frac{1}{2 \sqrt{\alpha}} \int_{1}^{x} \tilde{f}(\sigma)\left(e^{\sqrt{\alpha}(\sigma-x)}-e^{-\sqrt{\alpha}(\sigma-x)}\right) d \sigma
\end{aligned}
$$

Hence (10) by differentiation.

\subsection{Strategy for building an improved OBC}

As described in Section 2, we are now looking for a local high resolution approximation of the solution of (8), with the assumption that we have access to a global coarse approximation.

In order to compute a finite difference approximation of the solution of (8), the interval $[0,1]$ is discretized using a uniform mesh $\left(x_{i}\right)_{0 \leq i \leq 2 N}$ with $x_{i}=i h$ and $h=\frac{1}{2 N}$. We also consider the coarse mesh $\left(x_{2 i}\right)_{0 \leq i \leq N}$. We denote by $L_{H}$ (resp. $L_{h}$ ) the usual three-point discrete operator corresponding to (8) on the coarse (resp. fine) grid. These two operators are consistent with $\mathcal{L}$ and are second order accurate. 
Using the notations introduced in Section 2, let us assume that we are interested in obtaining an accurate solution $U^{\text {loc }}$ on a local domain $\left[0, x_{2 i_{0}}\right]$. This local solution $U^{\text {loc }}=\left(U_{0}^{\text {loc }}, U_{1}^{\text {loc }}, \cdots, U_{2 i_{0}-1}^{\text {loc }}, U_{2 i_{0}}^{\text {loc }}\right) \in \mathbb{R}^{2 i_{0}+1}$ will be obtained by solving

$$
\left\{\begin{array}{l}
L_{h} U^{\text {loc }}=f_{h} \\
U_{0}^{\text {loc }}=0
\end{array}\right.
$$

with some convenient boundary condition at $x=x_{2 i_{0}}$. Ideally this boundary condition should be such that $U^{\text {loc }}$ coincides with the reference solution $U^{\text {ref }}=$ $\left(U_{0}^{\text {ref }}, U_{1}^{\text {ref }}, \cdots, U_{2 N}^{\text {ref }}\right) \in \mathbb{R}^{2 N+1}$ defined by

$$
\left\{\begin{array}{l}
L_{h} U^{\text {ref }}=f_{h} \\
U_{0}^{\text {ref }}=0 \\
U_{2 N}^{\text {ref }}=0 .
\end{array}\right.
$$

As pointed out in Section 2, the boundary condition at $x=x_{2 i_{0}}$ must use some exterior information, provided by a less accurate solution $U^{\text {ext }}$. We will assume here that $U^{\text {ext }}=\left(U_{0}^{\text {ext }}, \cdots, U_{2 i}^{\text {ext }}, \cdots, U_{2 N}^{\text {ext }}\right) \in \mathbb{R}^{N+1}$ is a coarse grid approximation of $u$, which has been previously computed by solving

$$
\left\{\begin{array}{l}
L_{H} U^{\text {ext }}=f_{H} \\
U_{0}^{\text {ext }}=0 \\
U_{2 N}^{\text {ext }}=0 .
\end{array}\right.
$$

In order to find $B_{h}, B_{H}$ and $g$ that we will use in our generalized OBC, we have to find a relationship of the form of the ideal one (7). At the continuous level, such a relationship can be obtained by making $w=u^{\text {ref }}-u^{\text {ext }}$ and $\tilde{f}=\mathcal{L}\left(u^{\text {ref }}-u^{\text {ext }}\right)$ in Theorem 1. This provides indeed, at least theoretically, a boundary operator $\mathcal{B}$ and a correction term $p$ such that $\mathcal{B} u^{\text {ref }}=\mathcal{B} u^{\text {ext }}+p$. Our approach consists at the discrete level in introducing $I$, the linear interpolation operator from the coarse grid to the fine grid, and making $U^{\text {ref }}-I U^{\text {ext }}$ play the role of $w$, and $F_{h}=L_{h}\left(U^{\text {ref }}-I U^{\text {ext }}\right)$ play the role of $f$. Then we choose $B_{h}\left(\right.$ resp. $\left.B_{H}\right)$ to be a discretization of the continuous transparent boundary operator $\mathcal{B}$ on the fine (resp. coarse) grid, and $g$ to be an approximation of $p$. We now describe the discrete implementation of the different ingredients that we have proposed in the previous section for the generalized OBC (6).

Regarding the boundary operators $B_{h}$ and $B_{H}$, we have chosen two second order approximations of $\mathcal{B}$. Since $B_{H}$ is applied to $U^{\text {ext }}$ which is defined on both sides of the open boundary location $x_{2 i_{0}}$, we have used the standard second order centered scheme:

$$
B_{H} U^{\mathrm{ext}}=\frac{1}{4 h}\left(U_{2 i_{0}+2}^{\mathrm{ext}}-U_{2 i_{0}-2}^{\mathrm{ext}}\right)+\lambda U_{2 i_{0}}^{\mathrm{ext}} .
$$

The problem is different for $B_{h}$, since it is applied to $U^{\text {loc }}$ which is defined only left from $x_{2 i_{0}}$. Therefore we must use a one-sided scheme. Using the fact that, if $u$ is solution of $\mathcal{L} u=f$, then $u^{\prime}\left(x_{i}\right)$ can be approximated with second order accuracy by $\frac{1}{h}\left(U_{i}-U_{i-1}\right)+\frac{h}{2}\left(\alpha U_{i}-f\left(x_{i}\right)\right)$, we have chosen the scheme:

$$
B_{h} U^{\mathrm{loc}}=\frac{1}{h}\left(U_{2 i_{0}}^{\mathrm{loc}}-U_{2 i_{0}-1}^{\mathrm{loc}}\right)+\frac{h}{2}\left(\alpha U_{2 i_{0}}^{\mathrm{loc}}-f\left(x_{2 i_{0}}\right)\right)+\lambda U_{2 i_{0}}^{\mathrm{loc}} .
$$


The most complicated task is the approximation of the correction term $p$ occurring in (10). If $F_{h}=L_{h}\left(U^{\mathrm{ref}}-I U^{\mathrm{ext}}\right)$, Theorem 2 (see Appendix) gives the expansion $F_{h}=F+\mathcal{O}\left(h^{4}\right)$ and $p$ will be approximated by

$$
g_{h}=\frac{T_{h}(\tilde{F})}{z\left(x_{2 i_{0}}\right)}
$$

where $\left(\tilde{F}_{i}\right)_{2 i_{0} \leq i \leq 2 N}=\left(F_{i} z\left(x_{i}\right)\right)_{2 i_{0} \leq i \leq 2 N}$ and $T_{h}(Z)$ is the trapezoidal rule to integrate the vector $Z: T_{h}(Z)=h\left(Z_{2 i_{0}}+Z_{2 N}\right)+2 h \sum_{i=2 i_{0}+1}^{2 N-1} Z_{i}$.

Its approximation for small values of $h$ is given by $g_{h}=g+\mathcal{O}\left(h^{4}\right)$ with

$$
\begin{aligned}
g=\frac{h^{2}}{4 z\left(x_{2 i_{0}}\right)} & \left(\left[\frac{\alpha}{2 h}\left(U_{2 N}^{\mathrm{ext}}-U_{2 N-2}^{\mathrm{ext}}\right)+\alpha h\left(\alpha U_{2 N}^{\mathrm{ext}}-f\left(x_{2 N}\right)\right)-f^{\prime}\left(x_{2 N}\right)\right] z\left(x_{2 N}\right)\right. \\
+ & {\left[\alpha U_{2 N}^{\mathrm{ext}}-f\left(x_{2 N}\right)\right] z^{\prime}\left(x_{2 N}\right) } \\
- & {\left[\frac{\alpha}{4 h}\left(U_{2 i_{0}+2}^{\text {ext }}-U_{2 i_{0}-2}^{\text {ext }}\right)-f^{\prime}\left(x_{2 i_{0}}\right)\right] z\left(x_{2 i_{0}}\right)-\left[\alpha U_{2 i_{0}}^{\text {ext }}-f\left(x_{2 i_{0}}\right)\right] z^{\prime}\left(x_{2 i_{0}}\right) } \\
& \left.-\alpha^{2} T_{h}\left(\left(U_{2 i}^{\text {ext }} z\left(x_{2 i}\right)\right)_{i_{0} \leq i \leq N}\right)+\alpha T_{h}\left(\left(f\left(x_{2 i}\right) z\left(x_{2 i}\right)\right)_{i_{0} \leq i \leq N}\right)\right) .
\end{aligned}
$$

See Appendix for the detailed calculations leading to this approximation.

Finally a candidate for an efficient $\mathrm{OBC}$ is the generalized boundary condition (6) with $B_{H}, B_{h}$ and $g$ defined respectively in (14), (15) and (17).

Remark 1 In [1, 4], the equivalent of Theorem 1 is given at the discrete level and could be a priori used in our context. It would give an expression of $g$ computed on the fine exterior grid, leading to the exact OBC. However, because of the oscillating nature of $F$ (between $u^{\prime \prime}$ and $-u^{\prime \prime}$, see Theorem 2 in the Appendix), it is not straightforward to express this term on the coarse grid. With our strategy which consists in understanding the global behavior of the right hand side at the continuous level, we are free to discretize it on the coarse grid.

\subsection{Numerical results}

We will present now some numerical results in order to illustrate the actual performance of this generalized OBC. The experiments were conducted with $\alpha=10$, and with $f$ chosen such that $u(x)=\sin (2 \pi x)$ is the exact solution of (8). Three different locations of the open boundary were considered: $x_{\mathrm{loc}}=0.24$, 0.4 and 0.64 .

The generalized OBC has been compared to two other OBCs written in the usual form (4), i.e. without correction term. The first one is a simple Dirichlet condition $\left(B_{h}=B_{H}=\mathrm{Id}\right)$, while the second one uses the discretized transparent boundary operators (14) and (15), like the generalized OBC.

The errors with regard to the reference solution are displayed on Figure 2, for a mesh size $h=1 / 200$. Three main remarks emerge: (i) the OBCs without correction term hardly improve the results w.r.t. the external coarse resolution solution $U^{\text {ext }}$; (ii) the results are not systematically improved when transparent operators (with no correction term) are used instead of Dirichlet operator; (iii) the use of the correction term in the generalized OBC definitely improves the accuracy of the local solution, by several orders of magnitude. 

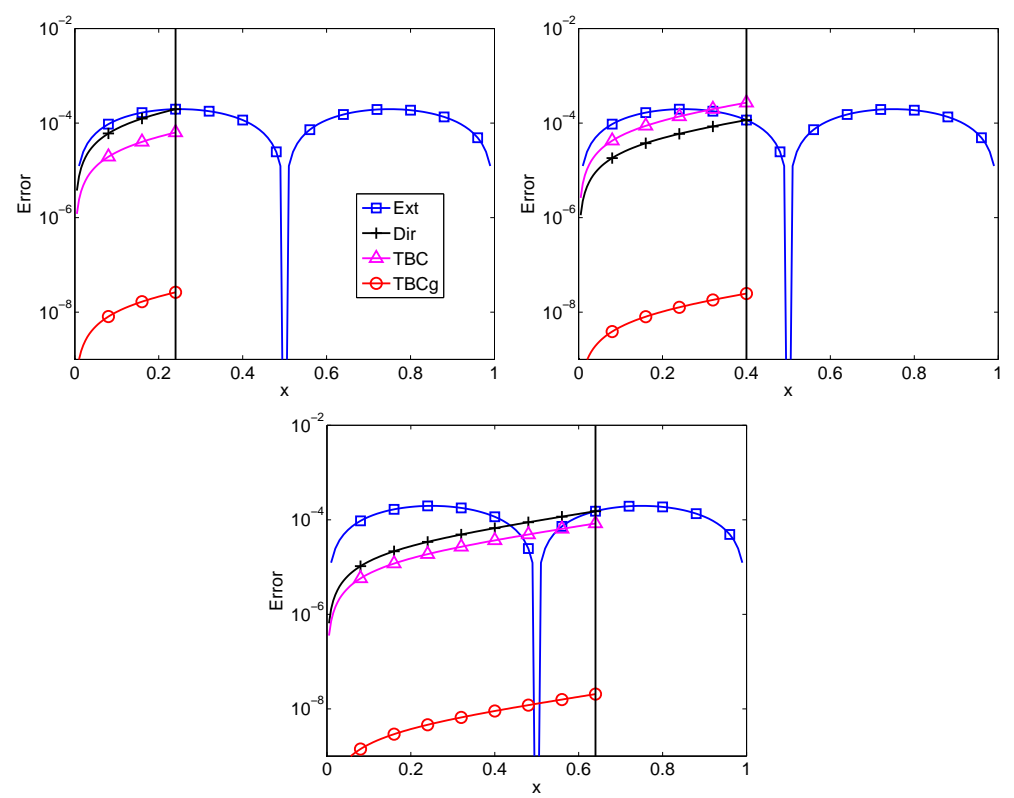

Figure 2: Error with regard to the reference solution for the external coarse resolution solution $\left(\left|U^{\text {ref }}-I U^{\text {ext }}\right|\right)($ Ext $)$ and for the local solution $\left(\left|U_{\mid \Omega^{\text {loc }}}^{\text {ref }}-U^{\text {loc }}\right|\right)$ computed with a Dirichlet OBC (Dir), a transparent OBC with no correction term $(\mathrm{TBC})$, and the generalized $\mathrm{OBC}(\mathrm{TBCg})$. The open boundary location is $x_{2 i_{0}}=0.24$ (top left), $x_{2 i_{0}}=0.4$ (top right) and $x_{2 i_{0}}=0.64$ (bottom).

To analyse more precisely the previous points, we have displayed the $L^{2}$ norms of the preceding errors in Figure 3 as a function of the mesh size $h$. We can notice important differences between the methods. As expected (due to the finite difference scheme) the error of the coarse resolution solution is $\mathcal{O}\left(h^{2}\right)$. The Dirichlet and transparent conditions with no correction term lead to an error which is also $\mathcal{O}\left(h^{2}\right)$, while the generalized OBC significantly improves the error estimate, which becomes $\mathcal{O}\left(h^{4}\right)$. Here is an explanation. Since the numerical scheme is second order, then $U_{2 i_{0}}^{\text {ext }}=u\left(x_{2 i_{0}}\right)+4 h^{2} c\left(x_{2 i_{0}}\right)+\mathcal{O}\left(h^{4}\right)$ and $U_{2 i_{0}}^{\mathrm{ref}}=u\left(x_{2 i_{0}}\right)+h^{2} c\left(x_{2 i_{0}}\right)+\mathcal{O}\left(h^{4}\right)$ for some continuous function $c$. As a consequence the exterior solution satisfies:

$B_{H} U^{\mathrm{ext}}=u^{\prime}\left(x_{2 i_{0}}\right)+\lambda u\left(x_{2 i_{0}}\right)+h^{2}\left(2 u^{(3)}\left(x_{2 i_{0}}\right) / 3+4 c^{\prime}\left(x_{2 i_{0}}\right)+4 \lambda c\left(x_{2 i_{0}}\right)\right)+\mathcal{O}\left(h^{4}\right)$, whereas the reference solution satisfies:

$B_{h} U^{\mathrm{ref}}=u^{\prime}\left(x_{2 i_{0}}\right)+\lambda u\left(x_{2 i_{0}}\right)+h^{2}\left(u^{(3)}\left(x_{2 i_{0}}\right) / 6+c^{\prime}\left(x_{2 i_{0}}\right)+\lambda c\left(x_{2 i_{0}}\right)\right)+\mathcal{O}\left(h^{4}\right)$.

Hence imposing the OBC : $B_{h} U^{\text {loc }}=B_{H} U^{\text {ext }}+g$ leads to an error which satisfies:

$$
B_{h}\left(U^{\mathrm{loc}}-U^{\mathrm{ref}}\right)=h^{2}\left(u^{(3)}\left(x_{2 i_{0}}\right) / 2+3 c^{\prime}\left(x_{2 i_{0}}\right)+3 \lambda c\left(x_{2 i_{0}}\right)\right)+g+\mathcal{O}\left(h^{4}\right) .
$$

Regarding the Dirichlet condition, the error at the interface is:

$$
U_{2 i_{0}}^{\text {loc }}-U_{2 i_{0}}^{\text {ref }}=3 h^{2} c\left(x_{2 i_{0}}\right)+\mathcal{O}\left(h^{4}\right) .
$$


Considering (18) and (19) we see that with both Dirichlet and transparent conditions without correction an error of order 2 in $h$ is introduced at the interface. However, in each case the magnitude of the error depends definitely on the solution $u$ and we have no assurance that the transparent $\mathrm{OBC}$ will yield a smaller error than the Dirichlet OBC. Regarding the generalized OBC with the correction term $g$, it absorbs the $h^{2}$ term, leading to an error of order 4 only on the boundary. This point is illustrated in Figure 4.

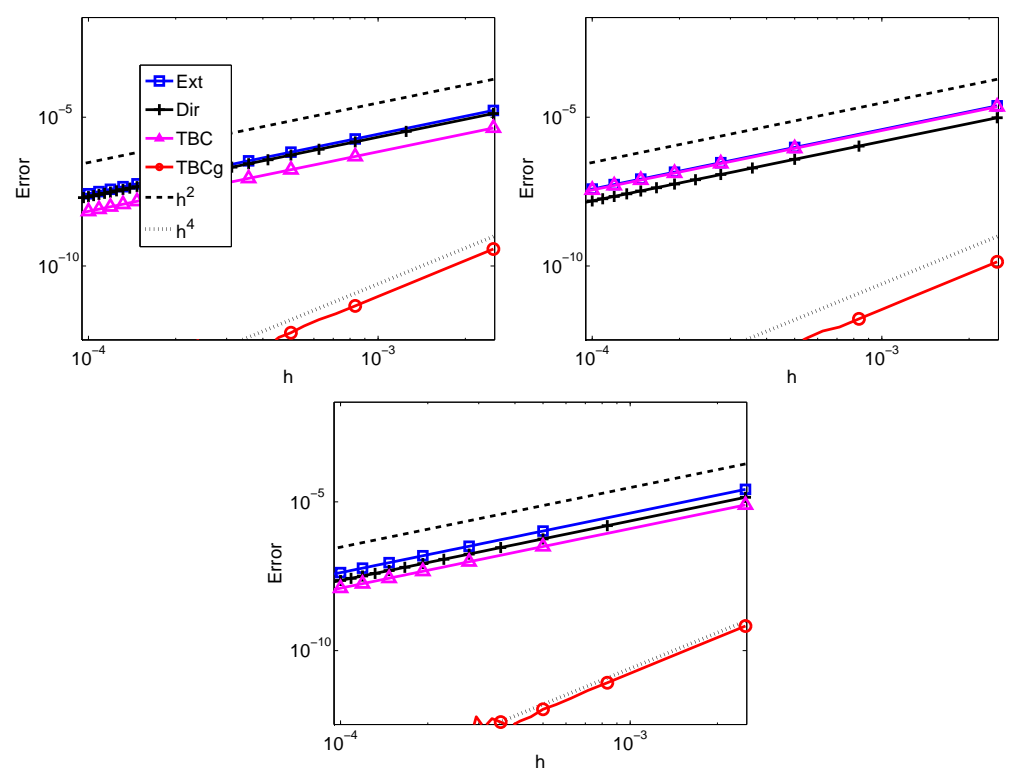

Figure 3: $L^{2}$ norm of the errors displayed in Figure 2 as a function of $h$. The open boundary is located at $x_{2 i_{0}}=0.24$ (top left), $x_{2 i_{0}}=0.4$ (top right) and $x_{2 i_{0}}=0.64$ (bottom).

\section{Generalized OBC using a Richardson extrap- olation}

The method presented in the previous section leads to very good performances of the generalized OBC. We were able to compute explicitly the correction term because of the simplicity of the equation, but this will no longer be the case for more complex equations. We therefore propose in the present section another way to get the correction term $g$ in (6), relying on Richardson extrapolation.

\subsection{Strategy to approximate an exact OBC}

Coming back to the preceding test case (8) described in Section 3, we consider now a Dirichlet condition, i.e. $B_{h} U^{\text {loc }}=U_{2 i_{0}}^{\text {loc }}$ and $B_{H} U^{\text {ext }}=U_{2 i_{0}}^{\text {ext }}$. Then, according to Lemma 1, we must choose $g=U_{2 i_{0}}^{\text {ref }}-U_{2 i_{0}}^{\text {ext }}$. Since $U^{\text {ref }}$ is unknown, this quantity cannot be directly computed, but we propose to approximate it by a Richardson extrapolation. 


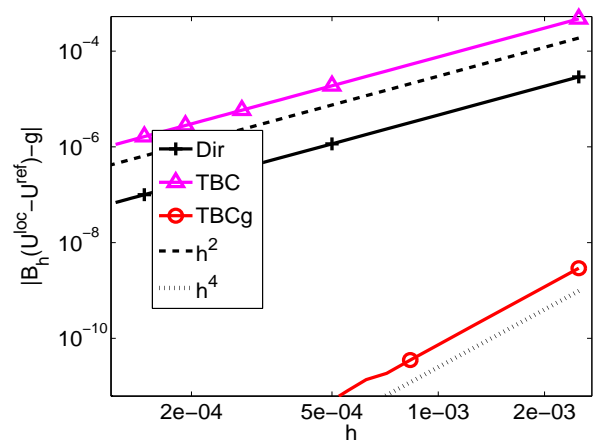

Figure 4: Residual $\left|B_{h}\left(U^{\text {loc }}-U^{\text {ref }}\right)-g\right|$ at point $x_{2 i_{0}}=0.4$ as a function of $h$ for the Dirichlet OBC (Dir), the transparent OBC with no correction term $(\mathrm{TBC})$ and the generalized $\mathrm{OBC}(\mathrm{TBCg})$.

The numerical scheme being second order accurate, we have:

$$
\begin{array}{r}
U_{i}^{\mathrm{ref}}=u\left(x_{i}\right)+h^{2} c\left(x_{i}\right)+\mathcal{O}\left(h^{4}\right) \\
U_{i}^{\text {ext }}=u\left(x_{i}\right)+4 h^{2} c\left(x_{i}\right)+\mathcal{O}\left(h^{4}\right)
\end{array}
$$

which yields $g=U_{2 i_{0}}^{\mathrm{ref}}-U_{2 i_{0}}^{\mathrm{ext}}=-3 h^{2} c\left(x_{2 i_{0}}\right)+\mathcal{O}\left(h^{4}\right)$. If $U^{\mathrm{EXT}}$ denotes the solution of the equation on an even coarser grid with mesh size $4 h$ (which can be computed at very low cost), then we have $U_{2 i_{0}}^{\text {ext }}-U_{2 i_{0}}^{\mathrm{EXT}}=-12 h^{2} c\left(x_{2 i_{0}}\right)+\mathcal{O}\left(h^{4}\right)$. This implies to approximate $g$ by $\left(U_{2 i_{0}}^{\text {ext }}-U_{2 i_{0}}^{\mathrm{EXT}}\right) / 4$. Hence the generalized OBC:

$$
U_{2 i_{0}}^{\text {loc }}=U_{2 i_{0}}^{\text {ext }}+\frac{1}{4}\left(U_{2 i_{0}}^{\text {ext }}-U_{2 i_{0}}^{\mathrm{EXT}}\right) .
$$

This very simple condition leads to very good performances w.r.t. previous OBCs, comparable to those obtained with the much more sophisticated generalized OBC derived in Section 3 (see Figure 5). Note that a figure similar to Figure 3 would show that the error with the reference solution also behaves like $\mathcal{O}\left(h^{4}\right)$.

\subsection{Application to a 1-D Shallow Water model}

In order to further investigate the preceding ideas, we will now consider a test case which is more complex in terms of the physics, but also closer to actual applications in oceanography or meteorology. We will use the 1-D linear shallow 

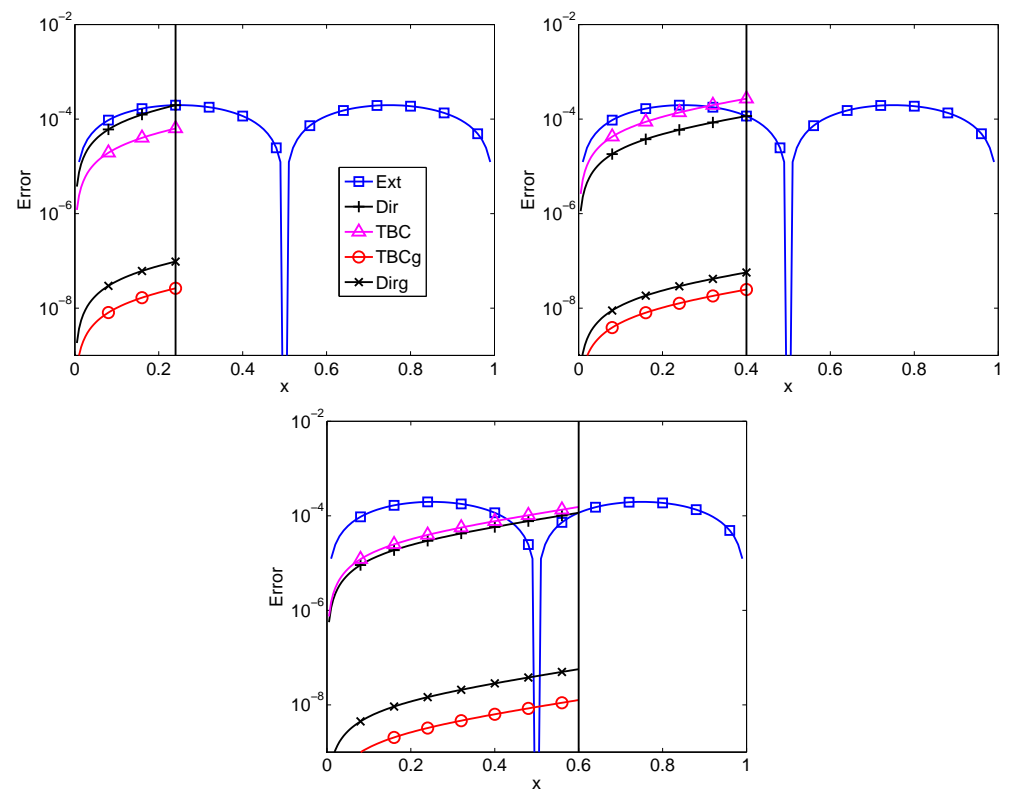

Figure 5: Same as Figure 2, but with the addition of condition (20) (labelled as Dirg).

water system:

$$
\begin{cases}\frac{\partial u}{\partial t}+U \frac{\partial u}{\partial x}+g \frac{\partial \eta}{\partial x}+r u=0 & \text { on }\left(-L_{1}, L_{2}\right) \times[0, T] \\ \frac{\partial \eta}{\partial t}+U \frac{\partial \eta}{\partial x}+D \frac{\partial u}{\partial x}+B u=0 & \text { on }\left(-L_{1}, L_{2}\right) \times[0, T] \\ \left(\sqrt{\frac{D}{g}} u+\eta\right)\left(-L_{1}, t\right)=0 & \text { for } t \in[0, T] \\ \left(\sqrt{\frac{D}{g}} u-\eta\right)\left(L_{2}, t\right)=0 & \text { for } t \in[0, T] \\ u(x, 0)=0 \text { and } \eta(x, 0)=\frac{1}{4+2 \cosh \left(\frac{x+5}{0.5}\right)} & \text { for } x \in\left(-L_{1}, L_{2}\right)\end{cases}
$$

where $U, D, g, r$ and $B$ are constants, corresponding respectively to a velocity scale, a depth scale, the gravity constant, a friction coefficient and a term related to the bottom topography gradient. This system is of hyperbolic nature, which is the dominant nature of ocean and atmosphere dynamics, and which strongly differs from the elliptic ODE considered in Section 3. We consider the usual regime where $|U| \ll c=\sqrt{g D}$, which means that the characteristic $w^{+}=$ $\frac{1}{2}(u \sqrt{D / g}+\eta)$ propagates eastward at the positive velocity $U+c$, and the characteristic $w^{-}=\frac{1}{2}(u \sqrt{D / g}-\eta)$ propagates westward at the negative velocity $U-c$. Note that this reference domain $\left(-L_{1}, L_{2}\right)$ is not closed, which explains our choice for the absorbing boundary conditions: $w^{+}\left(-L_{1}, t\right)=w^{-}\left(L_{2}, t\right)=$ $0, \forall t$. 
We chose the following values for the physical parameters: $L_{1}=20 \mathrm{~m}$, $L_{2}=10 \mathrm{~m}, D=25 \mathrm{~m}, g=10 \mathrm{~m} \cdot \mathrm{s}^{-2}, c=\sqrt{g D} \simeq 16 \mathrm{~m} \cdot \mathrm{s}^{-1}, U=0.1 \mathrm{~m} . \mathrm{s}^{-1}$, $r=3.10^{-3} \mathrm{~s}^{-1}$, and $B=0.095$. The open boundary is located at $x_{4 i_{0}}=-13$, as shown in Figure 6, which means that a wave enters the local domain $(-20,-13)$ during the experiment (approximately at $t=0.21$ ).
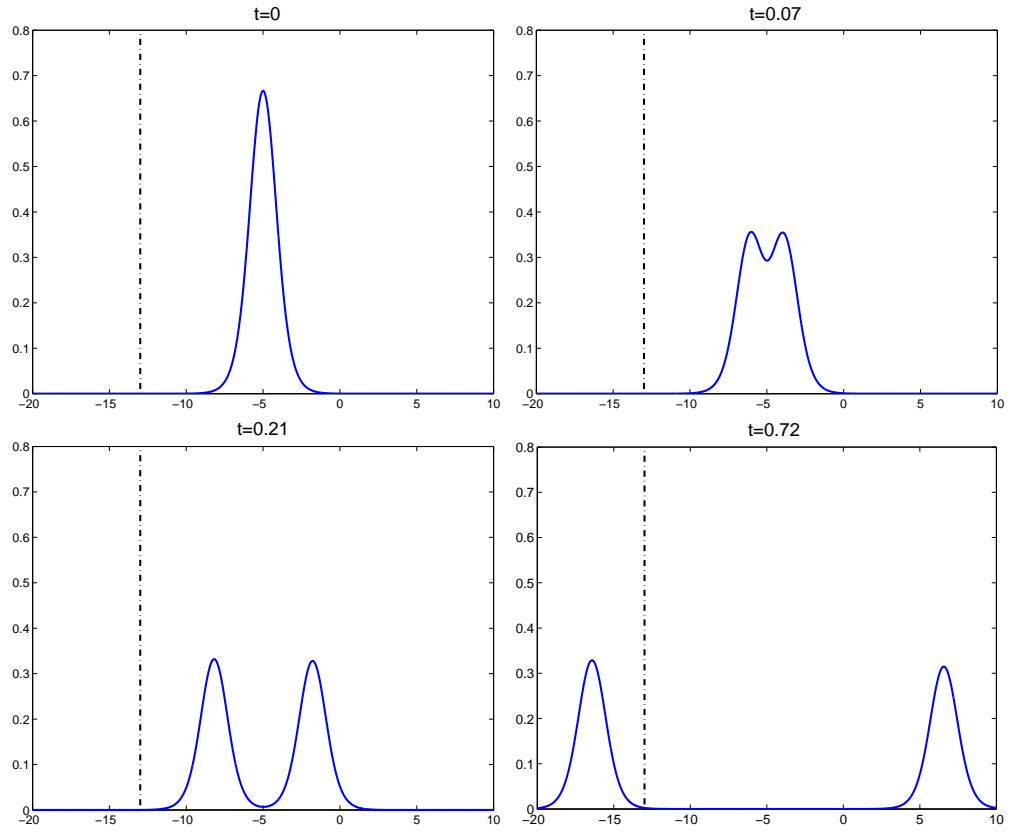

Figure 6: Solution of the shallow water model at successive instants $t=0$, $t=0.07, t=0.21$ and $t=0.72$.

We are now interested in the finite difference approximation of (21). The interval $\left(-L_{1}, L_{2}\right)$ is uniformly discretized with a mesh size $h:\left(x_{i}\right)_{0 \leq i \leq 4 N}$, while the time interval is discretized by a time step $d t:\left(t^{n}\right)_{0 \leq n \leq 4 M}$. Backward (resp. forward) Euler scheme is used on the characteristic variable $w^{+}$(resp. $w^{-}$) with the CFL condition $d t=h / \max (|U-c|,|U+c|)$. The solution which should be computed on this fine grid will be named $Z^{\text {ref }}=\left(U_{i, n}^{\text {ref }}, \eta_{i, n}^{\text {ref }}\right)_{0 \leq i \leq 4 N, 0 \leq n \leq 4 M}$, and is the reference solution. However, as described in the previous sections, we will solve the fine problem only on the local domain $\left(x_{i}\right)_{0 \leq i \leq 4 i_{0}} \times\left(t^{n}\right)_{0 \leq n \leq 4 M}$. This local solution will be named $Z^{\text {loc }}=\left(U_{i, n}^{\text {loc }}, \eta_{i, n}^{\text {loc }}\right)_{0 \leq i \leq 4 i_{0}, 0 \leq n \leq 4 M}$. Thus we need an $\mathrm{OBC}$ at $x_{4 i_{0}}=-13$. This boundary condition will use the coarse solution $Z^{\text {ext }}=\left(U_{2 i, 2 n}^{\text {ext }}, \eta_{2 i, 2 n}^{\text {ext }}\right)_{0 \leq i \leq 2 N, 0 \leq n \leq 2 M}$ which is computed on the coarse grid $\left(x_{2 i}\right)_{0 \leq i \leq 2 N} \times\left(t^{2 n}\right)_{0 \leq n \leq 2 M}$ (see Figure 7$)$.

We propose to implement the Richardson method presented in the previous section. The shallow water model (21) being of hyperbolic nature, we will consider boundary conditions dealing with the characteristic variables. Let $W_{4 i_{0}, n}^{-, \text {loc }}=U_{4 i_{0}, n}^{\text {loc }} \sqrt{D / g}-\eta_{4 i_{0}, n}^{\text {loc }}$ and $W_{4 i_{0}, n}^{-, \text {ext }}=U_{4 i_{0}, n}^{\text {ext }} \sqrt{D / g}-\eta_{4 i_{0}, n}^{\text {ext }}$ being the incoming characteristics from the point of view of the local domain. We propose the following generalized OBC:

$$
B_{h}^{n} Z^{\mathrm{loc}}=B_{H}^{n} Z^{\mathrm{ext}}+g^{n}, 0 \leq n \leq 4 M
$$




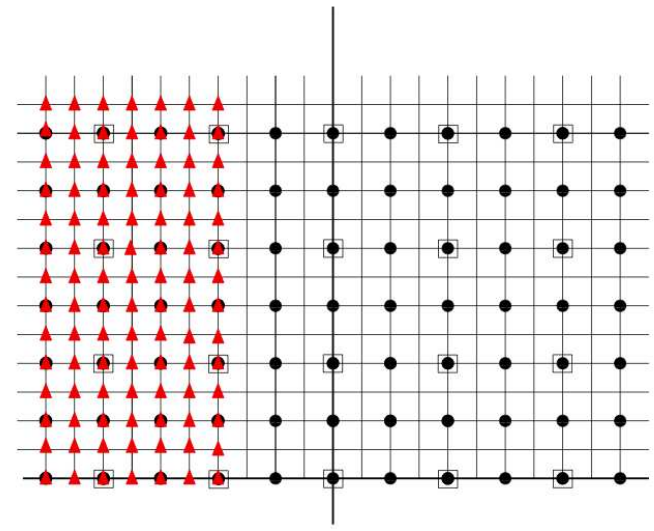

- Nodes for $\left(U^{\text {ext }}, \eta^{\text {ext }}\right)$

$\square$ Nodes for $\left(U^{\mathrm{EXT}}, \eta^{\mathrm{EXT}}\right)$

^ Nodes for $\left(U^{\text {loc }}, \eta^{\text {loc }}\right)$

Figure 7: Fine, coarse and very coarse meshes of $\left(-L_{1}, L_{2}\right) \times(0, T)$.

where

$$
\begin{aligned}
& B_{h}^{n} Z^{\mathrm{loc}}=W_{4 i_{0}, n}^{-, \text {loc }} \\
& B_{H}^{n} Z^{\mathrm{ext}}=\left\{\begin{array}{l}
W_{4 i_{0}, n}^{-, \text {ext }} \text { if } n \text { is even } \\
\frac{1}{2}\left(W_{4 i_{0}, n-1}^{-, \text {ext }}+W_{4 i_{0}, n+1}^{-, \text {ext }}\right) \text { if } n \text { is odd } .
\end{array}\right.
\end{aligned}
$$

The correction term $g^{n}$ must now be designed. Following Lemma 1, the expression of $g^{n}$ leading to the exact $\mathrm{OBC}$ is:

$$
g^{n}= \begin{cases}W_{4 i_{0}, n}^{-, \text {ref }}-W_{4 i_{0}, n}^{-, \text {ext }} & \text { if } n \text { is even } \\ W_{4 i_{0}, n}^{-, \text {ref }}-\frac{1}{2}\left(W_{4 i_{0}, n-1}^{-, \text {ext }}+W_{4 i_{0}, n+1}^{-, \text {ext }}\right) & \text { if } n \text { is odd }\end{cases}
$$

Since the scheme is first order accurate, we have the following consistency results:

$$
\begin{aligned}
& W_{4 i_{0}, n}^{-, \text {ref }}=w\left(x_{4 i_{0}}, t^{n}\right)+c_{1}\left(x_{4 i_{0}}, t^{n}\right) d t+c_{2}\left(x_{4 i_{0}}, t^{n}\right) h+\mathcal{O}\left(d t^{2}\right)+\mathcal{O}\left(h^{2}\right) \\
& W_{4 i_{0}, 2 n}^{-, \text {ext }}=w\left(x_{4 i_{0}}, t^{2 n}\right)+2 c_{1}\left(x_{4 i_{0}}, t^{2 n}\right) d t+2 c_{2}\left(x_{4 i_{0}}, t^{2 n}\right) h+\mathcal{O}\left(d t^{2}\right)+\mathcal{O}\left(h^{2}\right)
\end{aligned}
$$

where $c_{1}$ and $c_{2}$ are two continuous functions. Hence the behavior of $g^{n}$ for every $n$ :

$$
g^{n}=-c_{1}\left(x_{4 i_{0}}, t^{n}\right) d t-c_{2}\left(x_{4 i_{0}}, t^{n}\right) h+\mathcal{O}\left(d t^{2}\right)+\mathcal{O}\left(h^{2}\right) .
$$

This correction term will be approximated thanks to a Richardson extrapolation method using $Z^{\mathrm{EXT}}=\left(U_{4 i, 4 n}^{\mathrm{EXT}}, \eta_{4 i, 4 n}^{\mathrm{EXT}}\right)_{0 \leq i \leq N, 0 \leq n \leq M}$, the solution on the very coarse grid $\left(x_{4 i}\right)_{0 \leq i \leq N} \times\left(t^{4 n}\right)_{0 \leq n \leq M}$. It satisfies:

$$
W_{4 i_{0}, 4 n}^{-, \mathrm{EXT}}=w\left(x_{4 i_{0}}, t^{4 n}\right)+4 c_{1}\left(x_{4 i_{0}}, t^{4 n}\right) d t+4 c_{2}\left(x_{4 i_{0}}, t^{4 n}\right) h+\mathcal{O}\left(d t^{2}\right)+\mathcal{O}\left(h^{2}\right),
$$

and $g^{4 n}$ can be approximated by $\left(W_{4 i_{0}, 4 n}^{-, \text {ext }}-W_{4 i_{0}, 4 n}^{-, \mathrm{EXT}}\right) / 2$. At time indices $4 n+1$, 
$4 n+2$ and $4 n+3$, the correction term is approximated by interpolation:

$$
\begin{aligned}
g^{4 n} & \simeq \frac{1}{2}\left(W_{4 i_{0}, 4 n}^{-, \mathrm{ext}}-W_{4 i_{0}, 4 n}^{-, \mathrm{EXT}}\right) \\
g^{4 n+1} & \simeq \frac{1}{4}\left(W_{4 i_{0}, 4 n}^{-, \mathrm{ext}}+W_{4 i_{0}, 4 n+2}^{-, \mathrm{ext}}\right)-\frac{1}{8}\left(3 W_{4 i_{0}, 4 n}^{-, \mathrm{EXT}}+W_{4 i_{0}, 4 n+4}^{-, \mathrm{EXT}}\right) \\
g^{4 n+2} & \simeq \frac{1}{2} W_{4 i_{0}, 4 n+2}^{-, \mathrm{ext}}-\frac{1}{4}\left(W_{4 i_{0}, 4 n}^{-, \mathrm{EXT}}-W_{4 i_{0}, 4 n+4}^{-, \mathrm{EXT}}\right) \\
g^{4 n+3} & \simeq \frac{1}{4}\left(W_{4 i_{0}, 4 n+2}^{-, \mathrm{ext}}+W_{4 i_{0}, 4 n+4}^{-, \mathrm{ext}}\right)-\frac{1}{8}\left(W_{4 i_{0}, 4 n}^{-, \mathrm{EXT}}+3 W_{4 i_{0}, 4 n+4}^{-, \mathrm{EXT}}\right)
\end{aligned}
$$
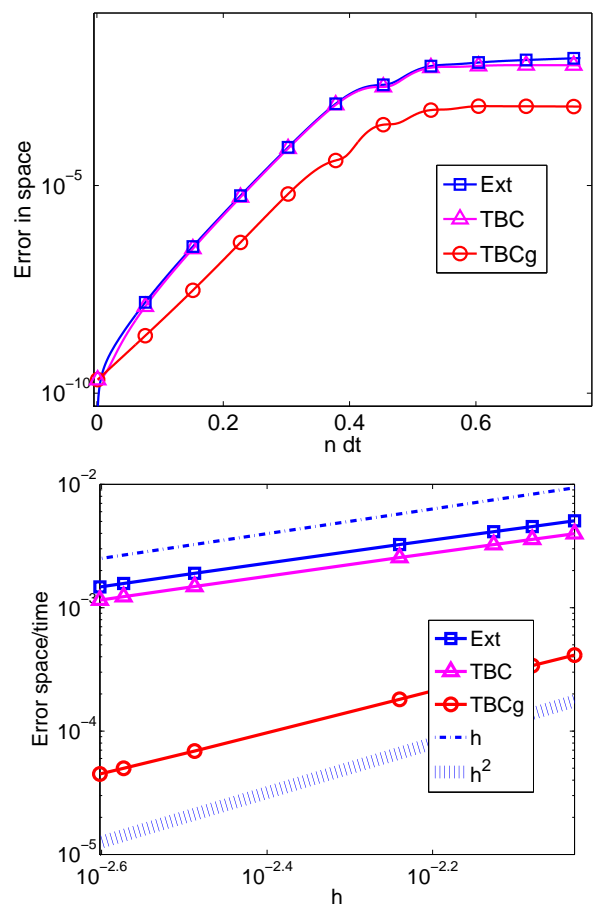

Figure 8: Left panel: errors $\left(\sum_{i=0}^{4 i_{0}} h\left|U_{i, n}^{\text {ref }}-I U_{i, n}^{\text {ext }}\right|^{2}\right)^{1 / 2}($ Ext $)$, and $\left(\sum_{i=0}^{4 i_{0}} h \mid U_{i, n}^{\text {ref }}-\right.$ $\left.\left.U_{i, n}^{\text {loc }}\right|^{2}\right)^{1 / 2}$ as a function of $n d t$ when (22) is used with $g^{n}=0$ (TBC) or with $g^{n}$ given by (23) (TBCg). Right panel: $\left(\sum_{n=0}^{4 M} \sum_{i=0}^{4 i_{0}} d t h\left|U_{i, n}^{\text {ref }}-I U_{i, n}^{\text {ext }}\right|^{2}\right)^{1 / 2}$ and $\left(\sum_{n=0}^{4 M} \sum_{i=0}^{4 i_{0}} d t h\left|U_{i, n}^{\mathrm{ref}}-U_{i, n}^{\mathrm{loc}}\right|^{2}\right)^{1 / 2}$ for (TBC) and (TBCg) as a function of $h$.

The improvement due to this correction term is evidenced in Figure 8. While the usual condition $\left((22)\right.$ with $\left.g^{n}=0\right)$ hardly improves the solution compared to the external coarse resolution solution, the error decreases by one order of magnitude when the corrected condition $\left((22)\right.$ with $g^{n}$ given by $\left.(23)\right)$ is used. Moreover the addition of this correction term makes the $L^{2}$ norm of the error in $\Omega^{\text {loc }} \times(0, T)$ change from $\mathcal{O}(h)$ (consistent with the order of the finite difference scheme) to $\mathcal{O}\left(h^{2}\right)$. 


\section{5 conclusion}

In many applications such as oceanography and meteorology, OBCs are usually written under the form $B_{h} U^{\text {loc }}=B_{H} U^{\text {ext }}$. The aim of this paper was to emphasize the interest in defining a generalized form $B_{h} U^{\text {loc }}=B_{H} U^{\text {ext }}+g, g$ being a correction term. For any $B_{h}$ and $B_{H}$ operators, if $g=B_{h} U^{\text {ref }}-B_{H} U^{\text {ext }}$, then $U^{\text {loc }}$ coincides with the reference solution. Obviously $U^{\text {ref }}$ is unknown and $g$ needs to be approximated. The obvious question is then to choose $B_{h}$ and $B_{H}$ such that a corresponding approximation of $g$ can be (as easily as possible) computed.

In this paper we have discussed two strategies. When $B_{h}$ and $B_{H}$ are discretizations of the transparent boundary operator, $g$ does not depend on $U^{\text {ref }}$. In that case we succeeded in getting an analytical expression and a good corresponding approximation for $g$ in the very simple case of the 1-D Laplace equation. Numerical experiments have underlined the good performance of this strategy. However, for more complex equations (e.g. 2-D case, non-linear equations), the transparent boundary operator cannot be used directly because of its non local nature, and therefore it must be approximated. This has consequences for $g$, which should be studied in a future work.

We also proposed to approximate $g$ numerically by a Richardson extrapolation method. In this case the simplest boundary condition operators $B_{h}$ and $B_{H}$ (Dirichlet for parabolic equations or incoming characteristic variables for hyperbolic equations) are convenient. Provided that several grid solutions with decreasing resolutions are available, such a strategy seems to improve the local solution significantly, at least in the simple 1-D test cases presented in this paper. The next step would be to implement a generalized OBC using this Richardson extrapolation strategy in a realistic oceanographic or atmospheric numerical model. Its actual performance will probably depend on the way the physical scales are resolved by the successive computation grids. Such an investigation will be the subject of a future paper.

\section{A Appendix: approximation of the correction term of the generalized OBC}

This appendix presents the detailed calculations leading to the approximation (17) for the correction term of the generalized OBC, in the test case described in Section 3. One has to provide an approximation for $p=\int_{x_{2 i_{0}}}^{1} R(\sigma) z(\sigma) d \sigma / z\left(x_{2 i_{0}}\right)$, the discretization of $R$ being $F_{h}=L_{h}\left(U^{\text {ref }}-I U^{\text {ext }}\right)$.

We need first to compute $F_{h}$. Let start by recalling some well-known properties for the finite difference operators $L_{h}$ and $L_{H}$.

Lemma 2 Let $f$ be in $C^{2}([0,1])$. Then $u$, solution of $(8)$, is in $\in C^{4}([0,1])$ and satisfies:

$$
\left(L_{h} \mathrm{~V}\right)_{i}=-u^{\prime \prime}\left(x_{i}\right)+\alpha u\left(x_{i}\right)-\frac{h^{2}}{12} u^{(4)}\left(x_{i}\right)+\mathcal{O}\left(h^{4}\right)
$$

where $\mathrm{V}=\left(u\left(x_{i}\right)\right)_{0 \leq i \leq 2 N}$. Moreover there exists $\mathrm{c}$ in $C^{2}([0,1])$ such that, if $U$ is solution of $L_{h} U=f_{h}$, then

$$
U_{i}-u\left(x_{i}\right)=h^{2} c\left(x_{i}\right)+\mathcal{O}\left(h^{4}\right) .
$$


Proof: The first result is obtained by Taylor expansion, while the second one is obtained thanks to properties of the inverse of the Laplacian matrix (e.g. [18] for more details).

Theorem 2 Let $f$ be in $C^{2}([0,1])$. Then the exterior error projected on the fine grid, $U^{\text {ref }}-I U^{\text {ext }}$, satisfies:

$$
L_{h}\left(U^{r e f}-I U^{e x t}\right)=F+\mathcal{O}\left(h^{4}\right)
$$

with $F=F^{1}+h^{2} F^{2}-\frac{h^{2}}{2} F^{3}$. The even and odd components of $F^{i}$ are defined by

$$
\begin{aligned}
& F^{1}=\left(\begin{array}{c}
\vdots \\
u^{\prime \prime}\left(x_{2 i}\right) \\
-u^{\prime \prime}\left(x_{2 i+1}\right) \\
\vdots
\end{array}\right), F^{2}=\left(\begin{array}{c}
\vdots \\
\frac{1}{3} u^{(4)}\left(x_{2 i}\right)+4 c^{\prime \prime}\left(x_{2 i}\right) \\
-\frac{1}{3} u^{(4)}\left(x_{2 i+1}\right)-4 c^{\prime \prime}\left(x_{2 i}\right) \\
\vdots
\end{array}\right) \\
& \text { and } F^{3}=\left(\begin{array}{c}
\vdots \\
0 \\
u^{(4)}\left(x_{2 i+1}\right)+f^{\prime \prime}\left(x_{2 i+1}\right) \\
\vdots
\end{array}\right) \text {, with c a continuous function. }
\end{aligned}
$$

Proof: Since $U^{\text {ref }}$ and $U^{\text {ext }}$ are solutions of (12) and (13), we obtain:

$$
\begin{aligned}
L_{h}\left(U^{\mathrm{ref}}-I U^{\mathrm{ext}}\right) & =f_{h}-I L_{H} U^{\mathrm{ext}}-L_{h} I U^{\mathrm{ext}}+I L_{H} U^{\mathrm{ext}} \\
& =f_{h}-I f_{H}+\left(I L_{H}-L_{h} I\right) U^{\mathrm{ext}} .
\end{aligned}
$$

The right hand side is the sum of two interpolation errors between the two grids: the first one measures the error on $f$ and the second one measures the error on the discrete operator.

Let us now estimate these errors. Computing $f_{h}-I f_{H}$ is straightforward:

$$
\left\{\begin{array}{l}
\left(f_{h}-I f_{H}\right)_{2 i}=f\left(x_{2 i}\right)-f\left(x_{2 i}\right)=0 \\
\left(f_{h}-I f_{H}\right)_{2 i+1}=f\left(x_{2 i+1}\right)-\frac{1}{2}\left(f\left(x_{2 i}\right)+f\left(x_{2 i+2}\right)\right)=-\frac{h^{2}}{2} f^{\prime \prime}\left(x_{2 i+1}\right)+\mathcal{O}\left(h^{4}\right) .
\end{array}\right.
$$

Let us now estimate $\left(I L_{H}-L_{h} I\right) U^{\text {ext }}$ for the even components. Relation (25) from Lemma 2 gives $U_{2 i}^{\text {ext }}=u\left(x_{2 i}\right)+4 h^{2} c\left(x_{2 i}\right)+\mathcal{O}\left(h^{4}\right)$ and we use (24) to obtain

$$
\begin{aligned}
{\left[\left(I L_{H}-L_{h} I\right) U^{\mathrm{ext}}\right]_{2 i} } & =-\frac{1}{(2 h)^{2}}\left(2 U_{2 i}^{\mathrm{ext}}-U_{2 i-2}^{\mathrm{ext}}-U_{2 i+2}^{\mathrm{ext}}\right) \\
& =+u^{\prime \prime}\left(x_{2 i}\right)+\frac{h^{2}}{3} u^{(4)}\left(x_{2 i}\right)+4 h^{2} c^{\prime \prime}\left(x_{2 i}\right)+\mathcal{O}\left(h^{4}\right) .
\end{aligned}
$$

Similarly the odd terms read:

$$
\begin{aligned}
{\left[\left(I L_{H}-L_{h} I\right) U^{\mathrm{ext}}\right]_{2 i+1} } & =\frac{\alpha}{2}\left(U_{2 i}^{\mathrm{ext}}+U_{2 i+2}^{\mathrm{ext}}\right)-\frac{1}{2(2 h)^{2}}\left(-2 U_{2 i}^{\mathrm{ext}}+U_{2 i-2}^{\mathrm{ext}}+U_{2 i+2}^{\mathrm{ext}}\right) \\
& -\frac{1}{2(2 h)^{2}}\left(-2 U_{2 i+2}^{\mathrm{ext}}+U_{2 i}^{\mathrm{ext}}+U_{2 i+4}^{\mathrm{ext}}\right) \\
& -\frac{\alpha}{2}\left(U_{2 i}^{\mathrm{ext}}+U_{2 i+2}^{\mathrm{ext}}\right)+\frac{1}{h^{2}}\left(-U_{2 i}^{\mathrm{ext}}-U_{2 i+2}^{\mathrm{ext}}+U_{2 i}^{\mathrm{ext}}+U_{2 i+2}^{\mathrm{ext}}\right) \\
& =-u^{\prime \prime}\left(x_{2 i+1}\right)-\frac{5 h^{2}}{6} u^{(4)}\left(x_{2 i+1}\right)-4 h^{2} c^{\prime \prime}\left(x_{2 i+1}\right)+\mathcal{O}\left(h^{4}\right) .
\end{aligned}
$$


Hence the result.

We now have to compute $T_{h}(\tilde{F})$ in (16) with $\tilde{F}_{i}=F_{i} z\left(x_{i}\right)$. This is the purpose of Theorem 3. But we first need preliminaries results to integrate $\tilde{F}$; vectors $\tilde{F}^{1}$ and $\tilde{F}^{2}$ have similar patterns: Lemma 3 explains how to integrate them, whereas $\tilde{F}^{3}$ is integrated thanks to Lemma 4 .

Lemma 3 Let $w$ be a $C^{4}([a, b])$ function and $\left(x_{i}\right)_{0 \leq i \leq 2 N}$ be a mesh of $[a, b]$ with $x_{i+1}-x_{i}=h$. Integrating $W=\left(w\left(x_{0}\right),-w\left(x_{1}\right), w\left(x_{2}\right), \cdots,-w\left(x_{2 N-1}\right), w\left(x_{2 N}\right)\right)$ by a trapezoidal rule gives $\frac{h^{2}}{4}\left(w^{\prime}\left(x_{2 N}\right)-w^{\prime}\left(x_{0}\right)\right)+\mathcal{O}\left(h^{3}\right)$.

Proof: Applying the trapezoidal rule on $W$ and then a Taylor expansion yields:

$$
\begin{aligned}
T_{h}(W)= & \frac{h}{2}\left(w\left(x_{0}\right)+w\left(x_{2 N}\right)\right)+h^{2} \sum_{i=1}^{N-1} \frac{w\left(x_{2 i}\right)-w\left(x_{2 i-1}\right)}{h}-h w\left(x_{2 N-1}\right) \\
= & \frac{h}{2}\left(w\left(x_{0}\right)+w\left(x_{2 N}\right)-2 w\left(x_{2 N-1}\right)\right)+h^{2} \sum_{i=1}^{N-1} w^{\prime}\left(x_{2 i}\right) \\
& -\frac{h^{3}}{2} \sum_{i=1}^{N-1} w^{\prime \prime}\left(x_{2 i}\right)+\mathcal{O}\left(h^{4}\right) .
\end{aligned}
$$

However the trapezoidal rule is second order accurate. Using it once again on the coarse grid we get for any $z \in C^{2}([a, b])$ :

$$
\int_{x_{0}}^{x_{2 N}} z(t) d t=\frac{2 h}{2}\left(z\left(x_{0}\right)+z\left(x_{2 N}\right)\right)+2 h \sum_{i=1}^{N-1} z\left(x_{2 i}\right)+\mathcal{O}\left(h^{2}\right) .
$$

As a consequence we have

$$
\begin{aligned}
2 h \sum_{i=1}^{N-1} w^{\prime}\left(x_{2 i}\right) & =\int_{x_{0}}^{x_{2 N}} w^{\prime}(t) d t-h\left(w^{\prime}\left(x_{0}\right)+w^{\prime}\left(x_{2 N}\right)\right)+\mathcal{O}\left(h^{2}\right) \\
& =w\left(x_{2 N}\right)-w\left(x_{0}\right)-h\left(w^{\prime}\left(x_{0}\right)+w^{\prime}\left(x_{2 N}\right)\right)+\mathcal{O}\left(h^{2}\right)
\end{aligned}
$$

and similarly

$$
2 h \sum_{i=1}^{N-1} w^{\prime \prime}\left(x_{2 i}\right)=w^{\prime}\left(x_{2 N}\right)-w^{\prime}\left(x_{0}\right)+\mathcal{O}(h) .
$$

We can now compute $T_{h}(W)$ :

$$
\begin{aligned}
T_{h}(W)= & \frac{h}{2}\left(w\left(x_{0}\right)+w\left(x_{2 N}\right)-2 w\left(x_{2 N-1}\right)\right)+\frac{h}{2}\left(w\left(x_{2 N}\right)-w\left(x_{0}\right)\right) \\
& -\frac{h^{2}}{2}\left(w^{\prime}\left(x_{0}\right)+w^{\prime}\left(x_{2 N}\right)\right)+\mathcal{O}\left(h^{3}\right)-\frac{h^{2}}{4}\left(w^{\prime}\left(x_{2 N}\right)-w^{\prime}\left(x_{0}\right)\right)+\mathcal{O}\left(h^{3}\right) \\
= & h^{2} \frac{w\left(x_{2 N}\right)-w\left(x_{2 N-1}\right)}{h}-\frac{h^{2}}{4}\left(w^{\prime}\left(x_{0}\right)+3 w^{\prime}\left(x_{2 N}\right)\right)+\mathcal{O}\left(h^{3}\right) \\
= & h^{2} w^{\prime}\left(x_{2 N}\right)-\frac{h^{2}}{4}\left(w^{\prime}\left(x_{0}\right)+3 w^{\prime}\left(x_{2 N}\right)\right)+\mathcal{O}\left(h^{3}\right) \\
= & \frac{h^{2}}{4}\left(w^{\prime}\left(x_{2 N}\right)-w^{\prime}\left(x_{0}\right)\right)+\mathcal{O}\left(h^{3}\right) .
\end{aligned}
$$


Lemma 4 Let $w$ be a $C^{4}([a, b])$ function and $\left(x_{i}\right)_{0<i<2 N}$ be a mesh of $[a, b]$ with $x_{i+1}-x_{i}=h$. Integrating $W=\left(0, w\left(x_{1}\right), 0, \cdots, w\left(x_{2 N-1}\right), 0\right)$ by the trapezoidal rule gives $\frac{1}{2} \int_{a}^{b} w(t) d t+\mathcal{O}(h)$.

Proof: The proof is similar to the one of Lemma 3.

We can now expand $g_{h}=T_{h}(\tilde{F}) / z\left(x_{2 i_{0}}\right)$ given in (16) for small values of $h$.

Theorem 3 The correction term $g_{h}$ given in (16) can be expanded as $g_{h}=$ $g+\mathcal{O}\left(h^{4}\right)$ with

$$
\begin{aligned}
g=\frac{h^{2}}{4 z\left(x_{2 i_{0}}\right)}( & \left(\left[\frac{\alpha}{2 h}\left(U_{2 N}^{e x t}-U_{2 N-2}^{e x t}\right)+\alpha h\left(\alpha U_{2 N}^{e x t}-f\left(x_{2 N}\right)\right)-f^{\prime}\left(x_{2 N}\right)\right] z\left(x_{2 N}\right)\right. \\
+ & {\left[\alpha U_{2 N}^{e x t}-f\left(x_{2 N}\right)\right] z^{\prime}\left(x_{2 N}\right) } \\
- & {\left[\frac{\alpha}{4 h}\left(U_{2 i_{0}+2}^{e x t}-U_{2 i_{0}-2}^{e x t}\right)-f^{\prime}\left(x_{2 i_{0}}\right)\right] z\left(x_{2 i_{0}}\right)-\left[\alpha U_{2 i_{0}}^{e x t}-f\left(x_{2 i_{0}}\right)\right] z^{\prime}\left(x_{2 i_{0}}\right) } \\
& \left.-\alpha^{2} T_{h}\left(\left(I U_{i}^{e x t} z\left(x_{i}\right)\right)_{2 i_{0} \leq i \leq N}\right)+\alpha T_{h}\left(\left(f\left(x_{i}\right) z\left(x_{i}\right)\right)_{2 i_{0} \leq i \leq N}\right)\right) .
\end{aligned}
$$

Proof: With obvious notations, we have $T_{h}(\tilde{F})=T_{h}\left(\tilde{F}^{1}\right)+\frac{h^{2}}{2} T_{h}\left(\tilde{F}^{2}\right)-\frac{h^{2}}{2} T_{h}\left(\tilde{F}^{3}\right)$. Using Lemmas 3 and 4, we obtain

$$
\begin{aligned}
& T_{h}\left(\tilde{F}_{1}\right)=\frac{h^{2}}{4}\left(\left(u^{\prime \prime} z\right)^{\prime}\left(x_{2 N}\right)-\left(u^{\prime \prime} z\right)^{\prime}\left(x_{0}\right)\right)+\mathcal{O}\left(h^{3}\right) \\
& T_{h}\left(\tilde{F}_{2}\right)=\mathcal{O}\left(h^{2}\right) \\
& T_{h}\left(\tilde{F}_{3}\right)=\frac{1}{2} \int_{x_{2 i_{0}}}^{1}\left(u^{(4)} z+f^{\prime \prime} z\right)(\sigma) d \sigma+\mathcal{O}(h)
\end{aligned}
$$

which implies

$$
\begin{aligned}
T_{h}(\tilde{F})= & \frac{h^{2}}{4}\left(\left(u^{(3)} z+u^{\prime \prime} z^{\prime}\right)\left(x_{2 N}\right)-\left(u^{(3)} z+u^{\prime \prime} z^{\prime}\right)\left(x_{2 i_{0}}\right)\right) \\
& -\frac{h^{2}}{4} \int_{x_{2 i_{0}}}^{1}\left(u^{(4)}+f^{\prime \prime}\right) z(\sigma) d \sigma+\mathcal{O}\left(h^{3}\right) .
\end{aligned}
$$

Since $u^{\prime \prime}=\alpha u-f$, we obtain:

$$
\begin{aligned}
T_{h}(\tilde{F})= & \frac{h^{2}}{4}\left(\left(\alpha u^{\prime}-f^{\prime}\right)\left(x_{2 N}\right) z\left(x_{2 N}\right)+(\alpha u-f)\left(x_{2 N}\right) z^{\prime}\left(x_{2 N}\right)\right) \\
& -\frac{h^{2}}{4}\left(\left(\alpha u^{\prime}-f^{\prime}\right)\left(x_{2 i_{0}}\right) z\left(x_{2 i_{0}}\right)+(\alpha u-f)\left(x_{2 i_{0}}\right) z^{\prime}\left(x_{2 i_{0}}\right)\right) \\
& -\frac{h^{2}}{4} \int_{x_{2 i_{0}}}^{1} \alpha(\alpha u-f)(\sigma) z(\sigma) d \sigma+\mathcal{O}\left(h^{3}\right) .
\end{aligned}
$$

Since on $\left(x_{2 i_{0}}, 1\right)$ we only know $U^{\text {ext }}, u$ will be approximated by $I U^{\text {ext }}$ in this formula, which leads to the result.

Acknowledgment. The authors would like to thank Prof. Robert Miller (Oregon State University) for his suggestions on possible improvements of the manuscript. 


\section{References}

[1] Arnold, A. Numerically absorbing boundary conditions for quantum evolution equations, VLSI Design 6 No. 1-4 (1998) 313-319.

[2] Antoine, X., Arnold, A., Besse, C., Ehrhardt, M. and Schädle, A. A review of transparent and artificial boundary conditions techniques for linear and nonlinear Schrödinger equations Commun. Comput. Phys. 4 (2008) no. 4, $729-796$.

[3] Blayo, E. and Debreu, L. Revisiting open boundary conditions from the point of view of characteristic variables. Ocean Modelling 9 (2005) no. 3, 231-252

[4] Ehrhardt, M. Discrete transparent boundary conditions for Schrödinger-type equations for non-compactly supported initial data. Appl. Numer. Math. 58 (2008), no. 5, 660-673.

[5] Engquist, B. and Majda, A. Absorbing boundary conditions for the numerical simulation of waves. Math. Comp. 31 (1977), no. 139, 629-651.

[6] Flather, R. A. A tidal model of the north-west European continental shelf. Mémoires Société Royale des Sciences de Liège. 10 (1976), no. 6, 141-164.

[7] Givoli, D. High order local non reflecting boundary conditions: a review. Wave Motion 39 (2004), 319-326.

[8] Hagstrom, T. Radiation boundary conditions for the numerical simulation of waves. Acta Numerica 8 (1999), 47-106.

[9] Lavelle, J.W. and Thacker, W.C. A pretty good sponge: Dealing with open boundaries in limited-area ocean models. Ocean Modelling 20 (2008), no.3, $270-292$.

[10] McDonald, A. Transparent boundary conditions for baroclinic waves: a study of two elementary systems of equations. Tellus A 57 (2005), 171-182.

[11] McDonald, A. Transparent lateral boundary conditions for baroclinic waves II. Introducing potential vorticity waves. Tellus A. 58 (2006), 210-220.

[12] McDonald, A. Transparent lateral boundary conditions for baroclinic waves III. Including vertical shear. Tellus A 61 (2009), 227-231.

[13] Mar-Or, A. and Givoli, D. High Order Global-Regional Model Interaction: Extension of Carpenter?s Scheme. Int. J. Numerical Methods in Engineering 77 (2009), 50-74.

[14] Marsaleix, P., Ulses, C., Pairaud, I., Herrmann, M.J., Floor, J.W., Estournel, C. and Auclair, F. Open boundary conditions for internal gravity wave modelling using polarization relations. Ocean Modelling 29 (2009), 27-42.

[15] Modave, A., Deleersnijder, E. and Delhez, E.J.M. On the parameters of absorbing layers for shallow water models. Ocean Dynamics 60 (2010), 6579 . 
[16] Neta, B., van Joolen, V., Dea, J.R. and Givoli, D. Application of Hhgh-order Higdon non-reflecting boundary conditions to linear shallow water models. Communications in Numerical Methods in Engineering 24 (2008), 1459 1466.

[17] Nycander, J., Mc Hogg, A. and Frankcombe, L.M. Open boundary conditions for nonlinear channel flow. Ocean Modelling 24 (2008), 108-121.

[18] Strikwerda, J. C. Finite difference schemes and partial differential equations. Society for Industrial and Applied Mathematics (SIAM), Philadelphia, PA, (2004).

[19] Tsynkhov, S. V. Numerical solutions of problems on unbounded domains. A review. Appl. Numer. Math. 27 (1998), 456-532. 